\title{
BMJ Open Understanding burn injuries in Aboriginal and Torres Strait Islander children: protocol for a prospective cohort study
}

Rebecca Q Ivers, ${ }^{1,2}$ Kate Hunter, ${ }^{1,3}$ Kathleen Clapham, ${ }^{4}$ Julieann Coombes, ${ }^{1}$ Sarah Fraser, ${ }^{2}$ Serigne Lo, ${ }^{1}$ Belinda Gabbe, ${ }^{5}$ Delia Hendrie, ${ }^{6}$ David Read, ${ }^{7}$ Roy Kimble, ${ }^{8}$ Anthony Sparnon, ${ }^{9}$ Kellie Stockton, ${ }^{8}$ Renee Simpson, ${ }^{7}$ Linda Quinn, ${ }^{9}$ Kurt Towers, ${ }^{10}$ Tom Potokar, ${ }^{11}$ Tamara Mackean, ${ }^{12}$ Julian Grant, ${ }^{2}$ Ronan A Lyons, ${ }^{13}$ Lindsey Jones, ${ }^{14}$ Sandra Eades, ${ }^{15}$ John Daniels, ${ }^{4}$ Andrew J A Holland ${ }^{14,16}$

To cite: Ivers $\mathrm{RQ}$, Hunter $\mathrm{K}$, Clapham K, et al. Understanding burn injuries in Aboriginal and Torres Strait Islander children: protocol for a prospective cohort study. BMJ Open 2015;5:e009826.

doi:10.1136/bmjopen-2015009826

- Prepublication history for this paper is available online To view these files please visit the journal online (http://dx.doi.org/10.1136/ bmjopen-2015-009826).

Received 27 August 2015 Revised 17 September 2015 Accepted 21 September 2015

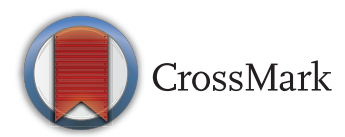

For numbered affiliations see end of article.

Correspondence to Professor Rebecca Ivers; rivers@george.org.au

\section{ABSTRACT}

Introduction: Although Aboriginal and Torres Strait Islander children in Australia have higher risk of burns compared with non-Aboriginal children, their access to burn care, particularly postdischarge care, is poorly understood, including the impact of care on functional outcomes. The objective of this study is to describe the burden of burns, access to care and functional outcomes in Aboriginal and Torres Strait Islander children in Australia, and develop appropriate models of care. Methods and analysis: All Aboriginal and Torres Strait Islander children aged under 16 years of age (and their families) presenting with a burn to a tertiary paediatric burn unit in 4 Australian States (New South Wales (NSW), Queensland, Northern Territory (NT), South Australia (SA)) will be invited to participate. Participants and carers will complete a baseline questionnaire; followups will be completed at 3, 6, 12 and 24 months. Data collected will include sociodemographic information; out of pocket costs; functional outcome; and measures of pain, itch and scarring. Health-related quality of life will be measured using the PedsQL, and impact of injury using the family impact scale. Clinical data and treatment will also be recorded. Around 225 participants will be recruited allowing complete data on around 130 children. Qualitative data collected by in-depth interviews with families, healthcare providers and policymakers will explore the impact of burn injury and outcomes on family life, needs of patients and barriers to healthcare; interviews with families will be conducted by experienced Aboriginal research staff using Indigenous methodologies. Health systems mapping will describe the provision of care.

Ethics and dissemination: The study has been approved by ethics committees in NSW, SA, NT and Queensland. Study results will be distributed to community members by study newsletters, meetings and via the website; to policymakers and clinicians via policy fora, presentations and publication in peer-reviewed journals.

\section{Strengths and limitations of this study}

- The study has support from peak Aboriginal health bodies, and data will be collected by Aboriginal research assistants or staff with significant experience working with Aboriginal people.

- The study will include detailed measures of cost of care as well as functional outcomes in Aboriginal and Torres Strait Islander children sustaining serious burns; data not previously collected.

- The unique collaboration between Indigenous and non-Indigenous researchers, clinical and policy stakeholders, and community members will ensure strong translation to practice.

- The study will only identify children who are treated at tertiary burn units.

- There is a high chance of loss to follow-up although this will be mitigated by data linkage.

\section{INTRODUCTION}

Burns are an important cause of injury in children in Australia. More than one-third of those affected by thermal injury in 2009-2010 were children aged 0-14 years, with $27 \%$ those aged $0-4$ years and $10 \%$ aged 5-14 years. The Australian Institute of Health and Welfare (AIHW) reported that in 2009-2010, there were 2220 children hospitalised for burn injury across Australia. Burns are serious injuries; $13.3 \%$ of all cases of burns have a high threat to life, ${ }^{1}$ with onequarter of hospitalised patients for burns found to have hospital stays of at least 1 week, testament to their serious nature. ${ }^{2}$

Aboriginal and Torres Strait Islander children are hospitalised for burns and scalds twice as often as for other children. ${ }^{3}$ 
Although there are few research studies or reports describing burns in Australian children, the Burns Registry of Australia and New Zealand shows that, of the patients admitted to Australian burn units, $11 \%$ of paediatric patients identified as Aboriginal or Torres Strait Islander in 2013-2014. ${ }^{4}$ The rate of burn injury per 100000 population was also substantially higher for Aboriginal and Torres Strait Islander people overall compared with the rest of the population at 14.4/100 $000 \mathrm{vs}$ $6.1 / 100000{ }^{1}$

As burns treatment itself can often be very painful and protracted, it is important to ensure families are adequately supported through what can be both a complex and traumatic process. While there are Aboriginal liaison units at most tertiary paediatric hospitals, it is unclear how well patients are supported with culturally appropriate and supportive care either in the hospital, or once they have left the hospital setting. Burn units report regularly losing contact with Aboriginal or Torres Strait Islander patients discharged to locations away from urban areas, although there has been no systematic analysis to assess how often this occurs. The accessibility of high-quality care to Aboriginal children, who constitute a high proportion of burn patients, particularly those from regional and remote settings, is particularly important, given the complexity of long-term burn care, where access to appropriate treatment is essential to produce good long-term outcomes. ${ }^{5}$

There are documented gaps in access by Aboriginal people to healthcare, both in primary care settings and in tertiary care. Aboriginal community-controlled health services are important in providing appropriate primary care, and there has been an emphasis on prioritising access to primary care of Aboriginal people to maximise prevention opportunities for chronic and other conditions. Nonetheless, there have been numerous studies highlighting the delays for Aboriginal people in accessing hospital care (suggestive of problems with the interface between primary care and the acute sector) for a variety of health conditions. ${ }^{6}$ Additionally, gaps in understanding of the care of Aboriginal people once they are discharged from hospital, including how they access medications prescribed and how the cost of prescribed care acts as a barrier to receipt of such care. ${ }^{6}$ Despite the development of Aboriginal liaison units in hospitals, while cultural security and cultural respect remain fundamental principles for Aboriginal Community Controlled Health Services (ACCHS), it is not clear how often these are achieved in other health services, which may inhibit their use by Aboriginal people.

\section{Measuring the outcomes and costs of burns}

Advances in all aspects of critical care and surgical technique have ensured that most children now survive their burn injury. Increasingly, therefore, the critical lens has moved from survival to morbidity, with one of the most important issues the prevention of long-term functional loss. The components that characterise long-term loss of function and disability in burn patients can include scarring, itch and pain, as well as a range of other elements including health-related quality of life, and psychosocial outcomes. There is, however, limited research evaluating such outcomes in children sustaining burns. The main studies are from US populations, ${ }^{7}$ which may have some relevance to the broader community in Australia, but are very unlikely to represent the experiences of Aboriginal or Torres Strait Islander children. In US populations, Sheridan $e t a l^{8}$ showed that in children surviving massive burns, factors that were related to better outcomes as measured by various domain scores on the 36-item Short Form Health Survey (SF-36) scale include family functional status, early reintegration into usual activities and consistent clinic visits. A recent study highlighted the importance of family characteristics on recovery from burns. ${ }^{9}$ Given that Aboriginal and Torres Strait Islander children are significantly over-represented in burns, are more likely to be living in out of home care ${ }^{10}$ and may experience difficulty in accessing healthcare services for a variety of reasons, understanding care received, functional outcomes and their relationship, are crucial to development and implementation of appropriate care.

Further, while there is a consensus that the cost of treating patients with burn injury is expensive, little is known about the true cost of a burn. ${ }^{11-13}$ In 1993-1994, burns were found to account for $2 \%$ of the total cost of injury, poisonings and musculoskeletal disorders. ${ }^{14}$ A later study investigated health system and total cost of injury in Western Australia by age: burn injured casualties of less than 20 years old accounted for $43 \%$ and $37 \%$, respectively, of the total cost of burn injuries. ${ }^{15}$ A recent Australian study ${ }^{12}$ found acute costs for an average adult burn patient were AU $\$ 71000$ with percentage of total body surface area (\%TBSA) injured the primary determinant of cost. This is likely to represent a small proportion of the total cost of burn injury and treatment, with burn injuries incurring significant longer term costs following initial acute admission. ${ }^{16}$ There is no research in Australia examining the cost of burns in Aboriginal children, or cost-effectiveness of various modes of treatment.

The objective of this study is to describe the burden of burns, access to care and functional outcomes in Aboriginal children with serious burns; in conjunction with burn clinicians, health services and Aboriginal community representatives, these data will be used to inform the development of appropriate, best practice, models of care. Specific aims are to: (1) describe the impact of burns in Aboriginal and Torres Strait Islander children; (2) describe the treatment received and its cost to health services and communities, and compare burn treatment to minimum clinical guidelines to identify gaps; (3) identify barriers to healthcare for Aboriginal and Torres Strait Islander children who sustain serious burns, from the perspective of the patient, their families 
and their health care providers, and document the extent to which these barriers contribute poor health outcomes; (4) inform the development of clear and practical clinical guidelines and a strategy for their use in health services and community settings.

\section{METHODS AND ANALYSIS}

\section{Eligible population and recruitment}

All children under 16 years presenting or referred for treatment for burns to participating tertiary burn units in the Australian states of New South Wales (NSW), Queensland, South Australia (SA) and the Northern Territory (NT) will be eligible for participation. All parents/carers will be asked whether the child is Indigenous using the Standard Indigenous Question ${ }^{17}$ at time of presentation at the burn unit as part of routine admission questions. Once survival of the child is assured, patient care teams will be consulted about the optimal time for recruitment into the study. Eligible families will be approached and given written and verbal information about the study during the first visit to the burn unit, prior to patient discharge. If the patient care team feels it appropriate, on this visit, eligible families will be asked to participate and complete a baseline interview. If recruitment is not possible on the first visit, families will be approached on the subsequent visit.

Consent will be obtained from parent or guardian for participation in the study. Children will also participate in the consent process if they are aged 12 years and above and the parent or guardian deems them able to participate in the consent process. Consent will also be requested to contact the usual medical provider at participants' places of residence or health practitioners who are involved in burn treatment subsequently and access burn-related medical records. Participants will also be asked to consent to primary care practitioners being involved in data collection, for example, in the case of those living in remote settings whereby local healthcare providers may be asked to administer follow-up questionnaires. Separate consent to access Medicare Benefit Scheme (MBS) and Pharmaceutical Benefit Scheme (PBS) records (allowing details of Government subsidised healthcare and pharmaceutical services utilised) will also be obtained.

Data will be collected using both quantitative and qualitative approaches. Data collection will include (1) participant interviews, (2) capture of clinical data from medical records and (3) linkage to MBS/PBS records. Additionally, to identify barriers and enablers to healthcare for Aboriginal and Torres Strait Islander children who sustain serious burns, from the perspective of the patient, their families and their healthcare providers, qualitative research will be conducted in a range of settings. Documentation of patient journeys, systems mapping of services and evaluation of health professionals' enactment of care will also be conducted. The information from all sources will be brought together with key stakeholders in policy roundtable sessions to inform discussion around development of appropriate models of care.

\section{Baseline interview}

Information collected at the baseline interview will include basic sociodemographic data (age, place of residence, measures of family structure, income and education), the burns first aid treatment (type, time from injury and duration), the timing of treatment, mode and timing of transportation to hospital, and preinjury quality of life. Tools used to measure sociodemographic characteristics, family disruption and family structure will be adapted from previous surveys including the NSW Population Health Survey ${ }^{18}$ and the Western Australian Aboriginal Child Health Survey, which developed and tested measures specifically for Aboriginal children and their families; ${ }^{19}$ clinical data will be extracted from patient medical records and preinjury quality of life measured using the Pediatric Quality of Life Inventory (PedsQL). ${ }^{20}$ To assess parental trauma (as the trauma experienced by the parent may influence care sought for the child), parents will also be asked to complete the Kessler 5 scale. ${ }^{21}$ Brief questions will also be asked about family disruption and travel related to treatment needs. Recruitment and baseline interviews will be conducted by trained Aboriginal or Torres Strait Islander research staff or by research staff with significant experience working with Aboriginal or Torres Strait Islander people.

\section{Follow-up interviews}

Follow-up interviews will be conducted at 3, 6, 12 and 24 months postinjury; the multiple measures are needed to determine recovery patterns and the more permanent consequences of injury. ${ }^{22}$ The interview will be conducted with the parents/carers. Data will be collected on care received (and out of pocket costs) since the last interview, health-related quality of life using the PedsQL,${ }^{20}$ pain, itch and activity limitations of the child as per baseline interviews, and return to school. If children are aged 5 years and over, they will also be asked to complete health-related quality of life measures, and measures of pain and itch. At annual interviews (12 and 24 months), parents/carers will also be asked to complete measures of psychological distress for themselves (Kessler 5), health-related quality of life measures for the child and brief questions on disruptions to family or employment due to treatment or caring needs. Details of outcomes measured are presented in table 1 .

\section{Clinical data}

The study research assistant at each site will extract information from clinical notes at regular intervals, including at baseline. This will include external cause and context of injury, operative procedures, outpatient visits, including number of visits to each service (eg, occupational therapist, psychologist, Aboriginal health worker, access to interpreter services), and scar management. In order 
Table 1 Outcomes collected at various time points

\begin{tabular}{|c|c|c|c|c|}
\hline Measure & Baseline & 3 months & 6 months & 12, 24 months \\
\hline \multirow[t]{7}{*}{ Interview } & Demographics & Treatment & Treatment & Treatment \\
\hline & $\mathrm{HRQOL}^{*}$ & $\mathrm{HRQOL}^{*}$ & $\mathrm{HRQOL}^{*}$ & $\mathrm{HRQOL}^{*}$ \\
\hline & Injury & Pain & Pain & Pain \\
\hline & Initial treatment & Itch & Itch & Itch \\
\hline & Kessler 5 & Family Disruption & Family Disruption & Family disruption \\
\hline & Family Demographics & Kessler 5 & Kessler 5 & Kessler 5 \\
\hline & Family function & POSAS† & POSAS† & POSAS† \\
\hline \multirow[t]{3}{*}{ Clinical data } & Hospital Medical & POSAS† & POSAS† & POSAS† \\
\hline & Records Datał & Hospital Medical & Hospital Medical & Hospital Medical \\
\hline & & Records Datał & Records Datał & Record Datał \\
\hline $\begin{array}{l}\text { *HRQOL: Healt } \\
\text { †POSAS: Patie } \\
\text { fHospital Medi } \\
\text { history, details } \\
\text { notes. }\end{array}$ & $\begin{array}{l}\text { yuality of life collected vi } \\
\text { server Scar Assessmen } \\
\text { Data. Injury: context, pr } \\
\text { ciplinary care, dressings }\end{array}$ & $\begin{array}{l}\text { e, including trans } \\
\text { e care admission }\end{array}$ & $\begin{array}{l}\text { ific events. Patien } \\
\text { for theatre and ac }\end{array}$ & $\begin{array}{l}\text { tory, medical } \\
\text { n assessment }\end{array}$ \\
\hline
\end{tabular}

to collect data on treatment, the research assistant, when appropriate, will have access to attend the weekly case conference meeting for each unit. Clinical information collected at baseline: date of burn, \% TBSA, depth of burn, location of burn (eg, hand, feet, face, genitals), ventilator days, surgical care (how much, timing, type), admission/length of stay to intensive care units, overall length of stay, complications (renal impairment, weight loss), where the patient is discharged to, type of care (use of splints, compression garments), assessment of activity limitation (from clinical notes) and measures of scarring using the Patient and Observer Scar Assessment Scale (POSAS) ${ }^{23}$ Clinical information will be collected at each follow-up visit or hospitalisation at the burn unit; if the patient follow-up interviews reveal care is received at other sites (eg, other hospitals), medical records will be accessed at those sites. Once the participant has agreed to participate in the study, a letter will be sent to the general practitioner and any allied health practitioners expected to be involved in follow-up care requesting cooperation in collection of study data including potential administration of questionnaires if required. At 6, 12 and 24 months from injury, a standard form (followed up by phone calls) will be sent to the participant's medical practitioner locally to request data on local clinical care, including services delivered by physiotherapists, nurses, counsellors or other allied health practitioners, and measures of patient health status if known.

We will also document the existing models of local and regional burn services in each study site. This will include mapping service provision at each site, documenting the model of care, from presentation to rehabilitation and follow-up care in the community. We will identify both structure-the organisation, communication, referral processes, rehabilitation and community outpatient care in place; and processes-the existence and use of structured care plans, clinical pathways, assessment protocols, rehabilitation prescriptions, and postdischarge management pathways in each site.

\section{Medicare linkage}

To access data on use of healthcare utilisation, participants will be asked for separate consent to link to MBS and PBS records. Items collected will include item numbers/description, including hospital and community items, type of specialty of provider, postcode of participant, postcode of provider and fee paid, including payments on Medicare-extended care plans which allow access to physiotherapy treatment and other allied health services. As, particularly in remote areas, a proportion of patients will be mobile, their Medicare records will allow ascertainment of location and frequency of treatment if contact with carers or participants is lost. Linkage will occur at the end of 24-month follow-up. Medicare is used to pay for most patient visits to medical practitioners in Aboriginal Medical Service settings, so this is a feasible way to track healthcare use, although it will not collect data on wound management, attendances at private practitioners. The proportion of visits missed will be checked by cross-referencing against patient interview data.

\section{Qualitative research}

In order to ascertain the impact of burn care and outcomes on family life, and understand needs of patients, and barriers to healthcare, including from the perspective of providers and policymakers, qualitative research is required. Qualitative research will be performed using Aboriginal ontology as a holistic framework that is based on interconnectedness, person-centred care and Aboriginal ways of knowing. ${ }^{24}$ Where required, the Aboriginal interpreter service will be employed for any families without English as a first language. Interviews will be audio recorded, transcribed in full and verified with participants to ensure trustworthiness of data. ${ }^{25}$

\section{Patient and family perspectives}

We will conduct semistructured interviews with individuals or small family groups, and a sample of families will be interviewed in each state. This will elicit feelings 
and reflections of the complexity of care experienced ${ }^{26}$ including the barriers to care and treatment, including components of treatment prescribed for use in community settings, such as use of compression garments. Families and their primary care providers at each site will be recruited by the research nurse and interviewed in the community until saturation of themes. Both urban and rural participants will be interviewed to identify issues relevant to each setting. Where qualitative interviews can generate rich contextual data, ${ }^{27}$ there is also a risk that data become a research commodity ${ }^{28}$ dissociated from cultural meanings. ${ }^{29}$ Particular care will be taken during interviews to ensure that the data remain connected to the larger moral community ${ }^{28}$ and are explicitly co-constructed between researcher and participants. ${ }^{30}$ Data will be transcribed and analysed thematically using content analysis. Detailed information on the participant's personal experience and factors influencing injury outcomes will be explored; results will be triangulated with questionnaire data to inform better understanding of factors impacting on outcomes.

\section{Clinical and stakeholder perspectives}

To better understand the barriers and enables at an institutional level, data will be collected following a three-tiered process to map patient journey, map health services and evaluate attitudes to care. In order to map patient journey, this study will utilise the Managing Two Worlds Together, Patient Journey Mapping Tool. ${ }^{31}$ Case study methodology will be used to map the actual patient journey of three Aboriginal or Torres Strait Islander children as they access health services following burn injury. The participants will be drawn from the overarching study. A narrative analytical approach will be used, and semistructured interviews with patients and their carers will be conducted asking questions about their experiences and the barriers and enablers to good care. The story will be written using Emden's core story creation narrative analysis and will use the relevant mapping tools as described in the Journey Mapping Tool. ${ }^{31}$ In order to map and describe the provision of care in health services enacted by health professionals, lead burn clinicians at each of the leading burn units in Australia will be consulted. During on-site consultation and observations, the lead burn clinicians will be asked in semistructured interviews to provide a description of typical patient burn care and pathways followed on presentation of a client with a burn injury. Health professionals and administrators involved in care along the patient pathways and state policy makers will also be consulted. Interviews will have a particular focus on system level approaches to care. The mapping will include both structure-the organisation, communication, referral processes, rehabilitation and community outpatient care in place; and processes-the existence and use of structured care plans, clinical pathways, assessment protocols, rehabilitation prescriptions and postdischarge management pathways in each site. To identify attitudes to care at an institutional level, including health professionals and policymakers involved in the management of burn injury, we will conduct a broad stakeholder consultation involving qualitative evaluation of knowledge, attitudes, practices and perceived needs of patients. The consultation will use in-depth semistructured interviews and will focus on management of burns, and perceived barriers to treatment, with a particular focus on system level approaches to establish a clear representation of how clinical guidelines are enacted.

All qualitative data will be analysed thematically, contextualised and triangulated to enable a better understanding of burn care for Aboriginal and Torres Strait Islander children.

\section{Sample size and analysis}

Retrieval of data from each site suggests that there are approximately 120 children presenting to or being referred to the burn units participating in this study each year, with between 10 and 20 each year for NT and SA units, and 40 and 50 for NSW and Queensland. However, given possible undercounting this may be as large as 150 or greater. Participants will be recruited until 225 participants have been recruited (over approximately 1820 months, expected conservative participation rate of at least $60 \%) .{ }^{32} \mathrm{We}$ anticipate collecting follow-up interview data for 2 years on approximately $70 \%$ of participants which will allow complete data on 130 children. ${ }^{32}$

We will document care received and describe patterns and characteristics of care by state, by place of residence, injury severity, and describe the proportions of children whose care meets standard clinical practice guidelines for medical management for paediatric burns. ${ }^{5}$ We will estimate the incidence of burn injuries in Aboriginal and Torres Strait Islander children presenting to burn units in each participating state, by extracting population counts for each state including age and gender distributions for Aboriginal and Torres Strait Islander people from the Australian Bureau of Statistics (ABS) Census data.

We will investigate the key predictors of health-related quality of life scores, and burn-related functional outcomes, including activity limitations, pain and return to school using random or mixed effects regression models. Measures to be assessed include age, gender, family function, type/amount of treatment received (including scar management/compression garments/ splints), type of patient (inpatient vs outpatient), place of residence (urban/rural) and psychological distress of parent and child. Although there is no literature describing predictors of PedsQL in children with burns, the following variables have been found to have an impact on the SF-36 score in paediatric patients with burns: age at injury, gender, functional family, early reintegration and consistent clinic visits; PedsQL scores have also been described in Australian children sustaining trauma, including burns. ${ }^{33}$ With 130 patients, four postbaseline measures at 3, 6, 12 and 24 months for each patient, correlation between two scores from the same participant is 
assumed to be $50 \%$, and assuming an exchangeable correlation matrix structure, we will have $80 \%$ power to determine a minimum absolute difference (increase or decrease) of $+/-0.3$ in global scores for each of the factors examined. The calculations are based on optitxs. $\mathrm{r}$ macro using R software V.3.1.3.

Linkage to MBS and PBS data will also provide important data on healthcare utilisation, particularly important where we may have significant loss to follow-up. We will examine healthcare utilisation by severity of burn, remoteness of place of residence and sociodemographic factors such as family structure, and age of child.

The cost analysis will adopt a health sector perspective and include costs borne by government, other agencies and families/carers. Costs will be assessed using a micro costing approach with all costs identified and valued for individual patients. Information on the use of health services will be collected from multiple sources including follow-up interviews with families/carers, clinical data and Medicare and PBS records. The cost of treating children with burn injuries will be calculated based on their resource use and the corresponding costs. These data will then be analysed using two approaches. First, a prevalence-based approach will be used to identify (1) the overall cost of treating burn injuries for the cohort of Aboriginal children and (2) costs disaggregated by cost component, subgroup and source of funds. Second, an individual-based approach will be adopted in which we will investigate associations between the mean individual cost and various factors including patient characteristics, healthcare utilisation and health outcomes using multiple linear regression techniques.

\section{Governance}

The study is managed by a project management committee, comprising the investigators and associate investigators, and research staff. This group has oversight of the study protocol, implementation and production of key outputs. An advisory group ensures community input into the study, and provides high level oversight of methods and relevance. This committee will ensure the processes used are cognisant of Indigenous understandings of health and well-being, that data analysis is based in an appropriate context and that meaningful dissemination and knowledge transfer activities occur. This will be chaired by an Indigenous member of the investigative team, and will include healthcare providers from primary care, including from Aboriginal Community Controlled Health Organisations as well as tertiary care providers and Aboriginal liaison units from the participating hospitals as well as the investigators and other state burn representatives. Community members will also be invited from each state.

\section{ETHICS AND DISSEMINATION}

Study results will be published in peer-reviewed journals presented at conferences and seminars; a study newsletter will be distributed regularly to participating families, community groups and Aboriginal peak bodies, and results will be disseminated via meetings, lay reports and via the study website. Policymakers, clinicians involved in treatment, pain management and rehabilitation in burn care (tertiary and community based), representatives of ACCHS and researchers will be invited to participate in policy roundtable discussions in years 1,3 and 4 to plan the study, review findings, discuss implications for policy development, and consider avenues and opportunities for implementation.

\section{IMPLICATIONS}

This study will investigate the previously understudied area of burns in Aboriginal and Torres Strait Islander children and identify the treatment received and its cost to health services and communities, and compare burn treatment to minimum clinical guidelines to identify gaps. Understanding what compromises cost-effective, clinically and culturally appropriate treatment for these injuries will substantially improve the evidence base and contribute to better care. We will quantify comprehensively the evidence practice gap on receipt of appropriate care and examine the impact on function; conduct a detailed barrier analysis of the underlying reasons for these gaps at the individual/ family, community, healthcare provider and health service levels; and establish a 'blueprint' for reform to overcome these barriers through engagement with communities, decision makers and other key stakeholders, guiding future intervention research. This study, with a combination of detailed interview, clinical and contextually rich qualitative data, offers a unique opportunity to generate, using a best practice, collaborative approach, important evidence to inform development of improved models of care for what is an over-represented and vulnerable population. Exploring the complexities of burn care for Aboriginal Australians will serve as a model for engaging health system reform that meets the needs of not only other disadvantaged groups in Australia, including people of low-income and of non-English speaking background, but also the broader population, as well as having important implications for burn care internationally.

\section{Author affiliations}

${ }^{1}$ Injury Division, The George Institute for Global Health, University of Sydney, Sydney, New South Wales, Australia

${ }^{2}$ School of Midwifery and Nursing, Flinders University, Adelaide, South Australia, Australia

${ }^{3}$ Poche Centre for Indigenous Health, University of Sydney, Sydney, New South Wales, Australia

${ }^{4}$ Australian Health Services Research Institute, University of Wollongong, Wollongong, New South Wales, Australia

${ }^{5}$ Department of Epidemiology and Preventive Medicine, Monash University, Melbourne, Victoria, Australia

${ }^{6}$ School of Public Health, Curtin University, Bentley, Western Australia, Australia

${ }^{7}$ National Critical Care \& Trauma Response Centre, Royal Darwin Hospital, Darwin, Northern Territory, Australia 
${ }^{8}$ Australian Centre for Children's Burns and Trauma Research, University of Queensland, Brisbane, Queensland, Australia

${ }^{9}$ Paediatric Burn Unit, Women's and Children's Hospital, Adelaide, South Australia, Australia

${ }^{10}$ Aboriginal Health, Northern and Central Adelaide Local Health Networks, Adelaide, South Australia, Australia

${ }^{11}$ Department of Burns \& Plastic Surgery, Welsh Centre for Burns \& Plastic Surgery, Swansea, UK

${ }^{12}$ Poche Centre for Indigenous Health and Wellbeing, Flinders University, Adelaide, South Australia, Australia

${ }^{13}$ Farr Institute, Swansea University Medical School, Wales, UK

${ }^{14}$ Faculty of Medicine, University of Sydney, Sydney, New South Wales, Australia

${ }^{15}$ Department of Aboriginal Health, Baker IDI Heart and Diabetes Institute, Melbourne, Victoria, Australia

${ }^{16}$ Discipline of Paediatrics and Child Health, The Children's Hospital at Westmead, Sydney, New South Wales, Australia

Twitter Follow Rebecca Ivers at @rebeccaivers

Contributors All authors were involved in conception of (RI, AJAH, KH, KC, RK, $\mathrm{DR}, \mathrm{AS}, \mathrm{SL}, \mathrm{BG}, \mathrm{TP}, \mathrm{KT}, \mathrm{RAL}, \mathrm{DH}, \mathrm{SE}, \mathrm{JD}$ ) or refining (KS, RS, LQ, JC, SF, TM, $J G, L J)$ of the study protocol. RI and LJ wrote the first draft of the manuscript; all authors contributed to editing and revisions of subsequent drafts.

Funding This work is supported by a project grant from the National Health and Medical Research Council of Australia, grant number APP1059038. RI and $B G$ are supported by NHMRC research fellowships. KH is supported by a fellowship from the Poche Centre for Indigenous Health, University of Sydney, and JC holds a research scholarship from the Lowitja Institute.

Competing interests None declared.

Ethics approval The study has been approved by ethics committees in each state, including the relevant Aboriginal health ethics bodies. These include the Human Research Ethics Committee of Northern Territory Department of Health and Menzies School of Health Research (EC00153); Central Australian Human Research Ethics Committee (EC00155); Aboriginal Health Research Ethics Committee (EC00185) (SA); Women's \& Children's Health Network Human Research Ethics Committee (EC00197) (SA); Aboriginal Health \& Medical Research Council of NSW Ethics Committee (EC00342); Sydney Children's Hospitals Network Human Research Ethics Committee (EC00130); The University of Queensland Medical Research Ethics Committee (EC00179); Children's Health Services Human Research Ethics Committee (EC00175) (QLD); Townsville Hospital and Health Service Human Research Ethics Committee (EC00183) (QLD); Department of Health Human Research Ethics Committee (EC00106) (National) and the Department of Human Services (for access to Medicare and Pharmaceutical benefits data).

Provenance and peer review Not commissioned; peer reviewed for ethical and funding approval prior to submission.

Open Access This is an Open Access article distributed in accordance with the Creative Commons Attribution Non Commercial (CC BY-NC 4.0) license, which permits others to distribute, remix, adapt, build upon this work noncommercially, and license their derivative works on different terms, provided the original work is properly cited and the use is non-commercial. See: http:// creativecommons.org/licenses/by-nc/4.0/

\section{REFERENCES}

1. Tovell A, McKenna K, Bradley C, et al. Hospital separations due to injury and poisoning, Australia 2009-10. Injury research and statistics series no. 69. Canberra: Australian Institute of Health and Welfare, 2012.

2. Wasiak J, Spinks A, Ashby K, et al. The epidemiology of burn injuries in an Australian setting, 2000-2006. Burns 2009:35:1124-32.

3. Australian Institute of Health and Welfare. The health and welfare of Australia's Aboriginal and Torres Strait Islander people, an overview 2011. Cat. no. IHW 42. Canberra: AlHW, 2011.

4. Burns Registry of Australia and New Zealand. Burns Registry of Australia and New Zealand annual report. 1st July 2013-30th June 2014. Melbourne: Monash University, 2014.
5. Kim LK, Martin HC, Holland AJ. Medical management of paediatric burn injuries: best practice. J Paediatr Child Health 2012;48:290-5

6. Scrimgeour M, Scrimgeour D. Health care access for Aboriginal and Torres Strait Islander people living in urban areas, and related research issues: a review of the literature. Darwin: Cooperative Research Centre for Aboriginal Health, 2007.

7. Tompkins RG, Liang MH, Lee AF, et al., Multi-Center Benchmarking Study Working G. The American Burn Association/Shriners Hospitals for Children Burn Outcomes Program: a progress report at 15 years. J Trauma Acute Care Surg 2012;73(3 Suppl 2):S173-8.

8. Sheridan RL, Hinson MI, Liang MH, et al. Long-term outcome of children surviving massive burns. JAMA 2000;283:69-73.

9. Sheridan RL, Lee AF, Kazis LE, et al. The effect of family characteristics on the recovery of burn injuries in children. $J$ Trauma Acute Care Surg 2012;73(3 Suppl 2):S205-12.

10. Australian Institute of Health and Welfare. Aboriginal and Torres Strait Islander child safety. Cat. no. IHW 50. Canberra: AlHW, 2011.

11. Dimick AR, Potts LH, Charles ED Jr, et al. The cost of burn care and implications for the future on quality of care. $J$ Trauma 1986:26:260-6.

12. Ahn C, Maitz P. The true cost of burn. Burns 2012;38:967-74.

13. Hemington-Gorse SJ, Potokar TS, Drew PJ, et al. Burn care costing: the Welsh experience. Burns 2009;35:378-82.

14. Mathers C, Penm R. Health system costs of injury, poisoning and musculoskeletal disorders in Australia 1993-94. AlHW cat. no. HWE 12: HWE Series no. 6. Canberra: Australian Institute of Health and Welfare, 1999

15. Hendrie D. The cost of injury in Western Australia during 2003: an overview of total and health system costs. Perth: Western Australian Government, 2005.

16. Sanchez JL, Bastida JL, Martínez MM, et al. Socio-economic cost and health-related quality of life of burn victims in Spain. Burns 2008;34:975-81.

17. Australian Institute of Health and Welfare. National best practice guidelines for collecting Indigenous status in health data sets. Cat no. IHW 29. Canberra: AlHW, 2010.

18. Centre for Epidemiology and Evidence. 2009-2010 Summary report from the New South Wales Child Health Survey. Sydney: NSW Ministry of Health, 2012.

19. Zubrick SR, Lawrence DM, Silburn SR, et al. The Western Australian Aboriginal Child Health Survey: the health of Aboriginal children and young people. Perth: Telethon Institute for Child Health Research, 2004.

20. Maskell J, Newcombe P, Martin G, et al. Psychosocial functioning differences in pediatric burn survivors compared with healthy norms. J Burn Care Res 2013;34:465-76.

21. Australian Institute of Health and Welfare. Measuring the social and emotional wellbeing of Aboriginal and Torres Strait Islander peoples. Cat. no. IHW 24. Canberra: AlHW, 2009.

22. Van Beeck EF, Larsen CF, Lyons RA, et al. Guidelines for the conduction of follow-up studies measuring injury-related disability. $J$ Trauma 2007:62:534-50.

23. Tyack Z, Simons M, Spinks A, et al. A systematic review of the quality of burn scar rating scales for clinical and research use. Burns 2012;38:6-18.

24. Martin K, Mirraboopa B. Ways of knowing, being and doing: a theoretical framework and methods for indigenous and indigenist re-search. J Aust Stud 2003;27:203-14.

25. Guba EG, Lincoln YS. Fourth generation evaluation. New York Sage Publications, 1989.

26. Liamputtong P, ed. Qualitative research methods. 3rd edn. Victoria: Oxford University Press, 2009.

27. Minichiello V, Aroni R. Indepth interviewing: principles, techniques, analysis. 3rd edn. Sydney: Pearson,Longman, 2008.

28. Denzin K. Qualitative inquiry under fire. California: Left Coast Press, 2009.

29. Tuhiwai Smith L. Decolonising methodologies: research and indigenous peoples. London: Zed Books, 2001.

30. Gubrium J, Holstein J. From the individual interview to the interview society. Postmodern interviewing. Thousand Oaks: Sage Publications, 2003:21-49.

31. Kelly J, Dwyer J, Pekarsky B, et al. Managing Two Worlds Together: stage 2-Patient Journey Mapping Tools. Melbourne: Lowitja Institute, 2012.

32. De Young AC, Kenardy JA, Cobham VE, et al. Prevalence, comorbidity and course of trauma reactions in young burn-injured children. J Child Psychol Psychiatry 2012;53:56-63.

33. Gabbe BJ, Simpson PM, Sutherland AM, et al. Functional and health-related quality of life outcomes after pediatric trauma. J Trauma 2011;70:1532-8. 